\title{
HI IN HII-GALAXIES AND THEIR COMPANIONS
}

\author{
E. BRINKS, C. L. TAYLOR AND D. L. THOMAS \\ NRAO, P.O. Box O, Socorro, NM 87801, USA \\ AND \\ E. D. SKILLMAN \\ Univ. Minnesota, Minneapolis, MN 55455, USA
}

\section{Introduction and Results}

HII galaxies are dwarf galaxies which are currently actively forming stars. We speculated that an interaction with an optically faint, but gas-rich object might be responsible for their enhanced star formation. This prompted us to search for companions with the VLA in the 21-cm line of HI. This has several advantages over optical searches (Campos-Aguilar et al. 1991, AJ, 106, 1784; Telles \& Terlevich 1995, MNRAS, 275, 1), e.g., the direct availability of redshifts.

The sample which we observed is volume limited $\left(\mathrm{V}_{\odot}<2500 \mathrm{~km} \mathrm{~s}^{-1}\right)$, and is based on HII galaxies with $\mathrm{M}_{\mathrm{B}}>-19 \mathrm{mag}$; it is drawn from the lists of Salzer et al. (1989, ApJS, 70, $447 \& 479)$. We detected 20 out of 21 objects observed with the VLA in its D-array (45" resolution) configuration (Taylor et al. 1995, ApJS, 99, 427). We searched for HI companions within 30 arcmin, and within a velocity range of $\pm 250 \mathrm{~km} \mathrm{~s}^{-1}$. Some $60 \%$ of the target galaxies have a companion (or are binary systems) which supports our working hypothesis that interactions might be the cause for the active star formation we see in the HII galaxies.

We have started a survey similar to the one described above, but on a sample of Low Surface Brightness (LSB) dwarf galaxies drawn from lists by Schombert et al. (1992, AJ, 103, 1107) and provided to us by Bothun (priv. comm.). These objects serve as a control sample for the HII galaxy observations. A first pass through our 18 LSB dwarf galaxies shows that only four of them have a companion, confirming our working hypothesis. 